\title{
Analysis of Building Construction Materials and Environmental Technology of Labrang Monastery
}

\author{
Qi Gao ${ }^{1, a^{*}}$, Xiangwu Meng, ${ }^{2, b}$, Daiwei Luo ${ }^{3, c}$ and J.W.R. Whitehand ${ }^{4, d}$ \\ ${ }^{1}$ Weifang University, Weifang, 261000, China \\ ${ }^{2}$ LanZhou University Of Technology, 730000, China \\ ${ }^{3}$ Suzhou University of Science and Technology, 215011, China \\ ${ }^{4}$ University of Birmingham, B15 2TT, United Kingdom
}

a564048577@qq.com/wfxygq@wfu.edu.cn, b84666097@qq.com, c441322693@qa.com, dWhoisldw@gmail.com

\section{Keywords: Tibetan Buddhist monastery; Labrang Monastery; Building} materials; Building environment technology

\begin{abstract}
Labrang Monastery is the only main monastery of the Gely Gec. Tibetan suddhism in Gannan area. From the perspective of building materials and t tnology, p per carries out literature research and fieldwork in order to comb and summariz the in constry cion of monastery
\end{abstract} buildings and common building materials. Furthermore, it st matica analyses the architectural phisics, (acoustics, optics and thermotics) of monaster wuilaings via thods such as research, servey and data analysis. These above have positive refe ntial value for the protection and renovation of Labrang Monastery and the modern design of region religious by ldings as well.

With the background of "One Belt and One Road" ro policy, the development of Labrang Monastery is confronted with new challenges ar mortuniturus a typical representative of religious buildings in Gannan area. It needs the solid basic ors matical study to explore how to protect and inherit regional architectural features and how to $\mathrm{id}$ the influence of cultural convergence.

At present, the research results of ang Mo astery abroad are relatively few, and mainly are from the religious and sociologi per, ectives, ocusing on the interpretation of Sino-Tibetan relations, politicians and relig us 11 as customs and cultures, rarely involving the Labrang Monastery the buil 'ng itself $\mathrm{J}$. Correspondingly, the domestic research is relatively rich, among which the resear in the field architecture mainly focuses on monastery morphology architectural space layo arch tural style, architectural art and architectural culture, while as for construction, or otb $\mathrm{r}$ aspects of th ong Monastery building materials, construction and physical environment tec ology search are not systematic, with only a few buildings once mentioned about. Labrang Monasu a typ al representative of Tibetan Buddhist architecture, which is well preserved ${ }^{-o m}$ th nersp ctive of materials and environmental technology, it has positive signific nce deepen the protection and inheritance system.

\section{Genera. troduction of Labrang Monastery}

Labrang My nastery was built in the reign of Kangxi in the Qing Dynasty (1708 A.D.)[4]. It is located on the west side of Xiahe County, which belongs to Gansu Province. Labrang Monastery is located near the base of Tea-Horse Trade. Under the influence of multi-culture, the monastery shows strong regional characteristics[5]. At present, the monastery is the key cultural relic protection unit in China and it is also one of the most perfect Tibetan Buddhism teaching system[6].

The whole monastery is facing south, surrounded by mountains and rivers which is very magnificent (Fig. 1). The north side of the monastery is Woxiang Mountain, and the south side is adjacent to Daxia River. The monastery covers an area of 866,000 square meters, composing 6 Sutra halls, 16 Buddhist temples and 28 living Buddha palaces. In addition, there are a large number of adjunct buildings such as lecture platform, monk's house and so on. Architecture layout conforms to 
the terrain, arranged according to different levels, architectural style has Tibetan and Han-Tibetan combination, architectural form belongs to the typical Gelug model of Tibetan Buddhism.

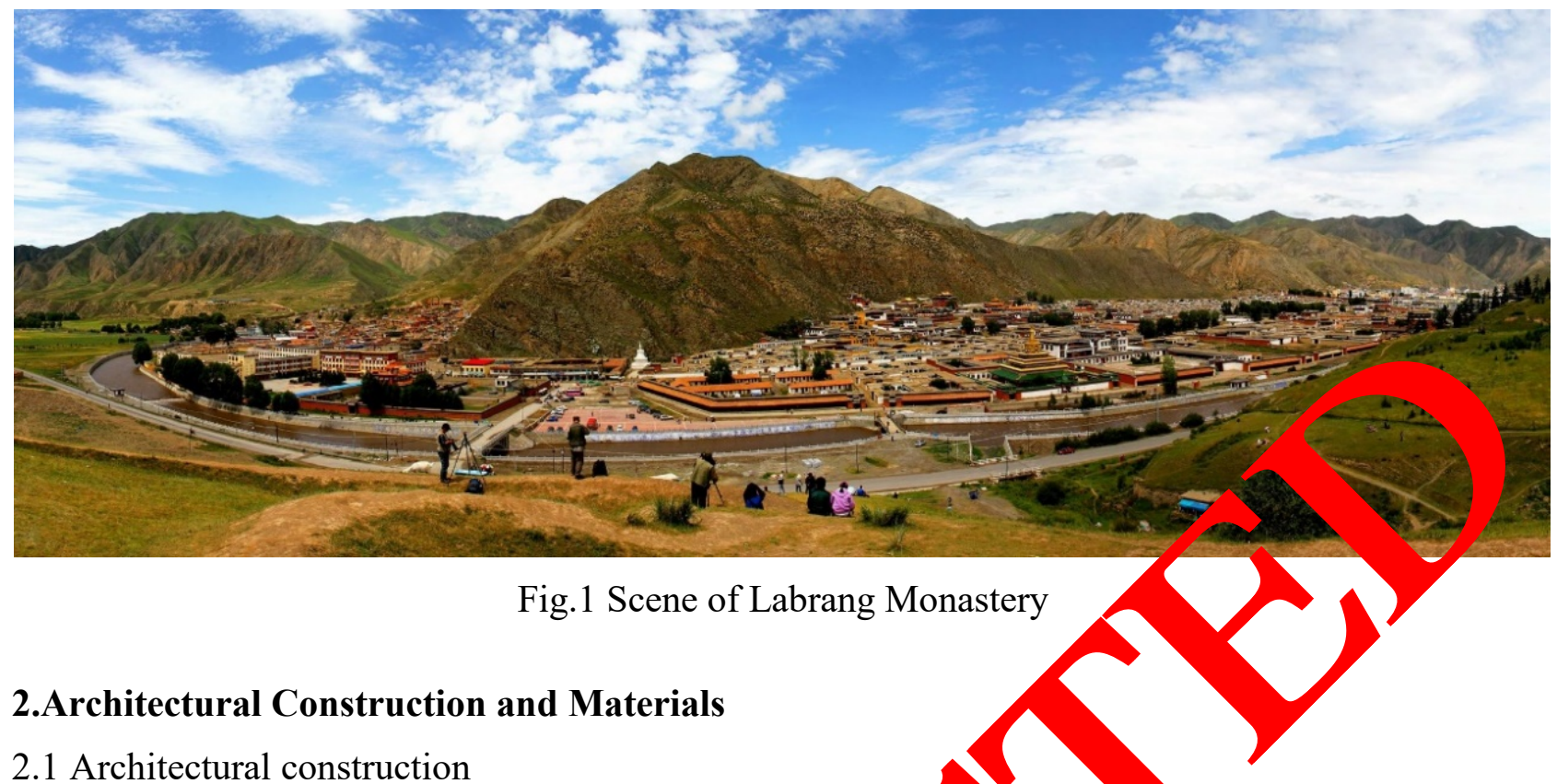

The architectural construction of Labrang Monastery ma ny includes 2 s walls and roofs. The wall part focus on the construction of stone wall and das bhora wall and the roof part mainly refers to Tibetan-style roof construction.

2.1.1 Wall construction

(1) the flake-stone wall construction.

Walls of stone are usually used in buildings buildings or the Sino-Tibetan style buildings, and th flaky stone walls have the supporting function as well. To the stone wall, its constru starts fi $m$ the top of the base of the wall foundation, and the bottom of the foundation is tar ed $\mathrm{w}$ silty cl After Tamping, the foundation groove is built with stone and loess mud, and th w w and The foundation groove is built to the ground, and then the stone w al contin to be built up. The bottom layer of the wall is 1.5-2 meters thick. The inner and outer on the of the made of flat Piece of stone, and the interior is filled with irregular slabs th. are s rimposed on each other. Repeated masonry like this, the stones overlap each other $y / 3$. The th ness of the wall is thicker at the bottom, but getting gradually thinner to the to From the wall p-ofile, it can be more clearly seen that the inner side of the wall remains vertical th outer de is inclined, which the angle of contraction is generally 6-7 degrees. When layino wall wo or nore layers, the upper wall is still thinner than the lower layer, and there is a pull $1 \mathrm{~g} \mathrm{~b}$ on the as well. The bonding material of the stone is the local black soil, which is mi into lurry. Tbe outer surface of the wall is painted white, red or yellow, according to the building auc, an the inner surface is generally painted white. (Fig.2) 


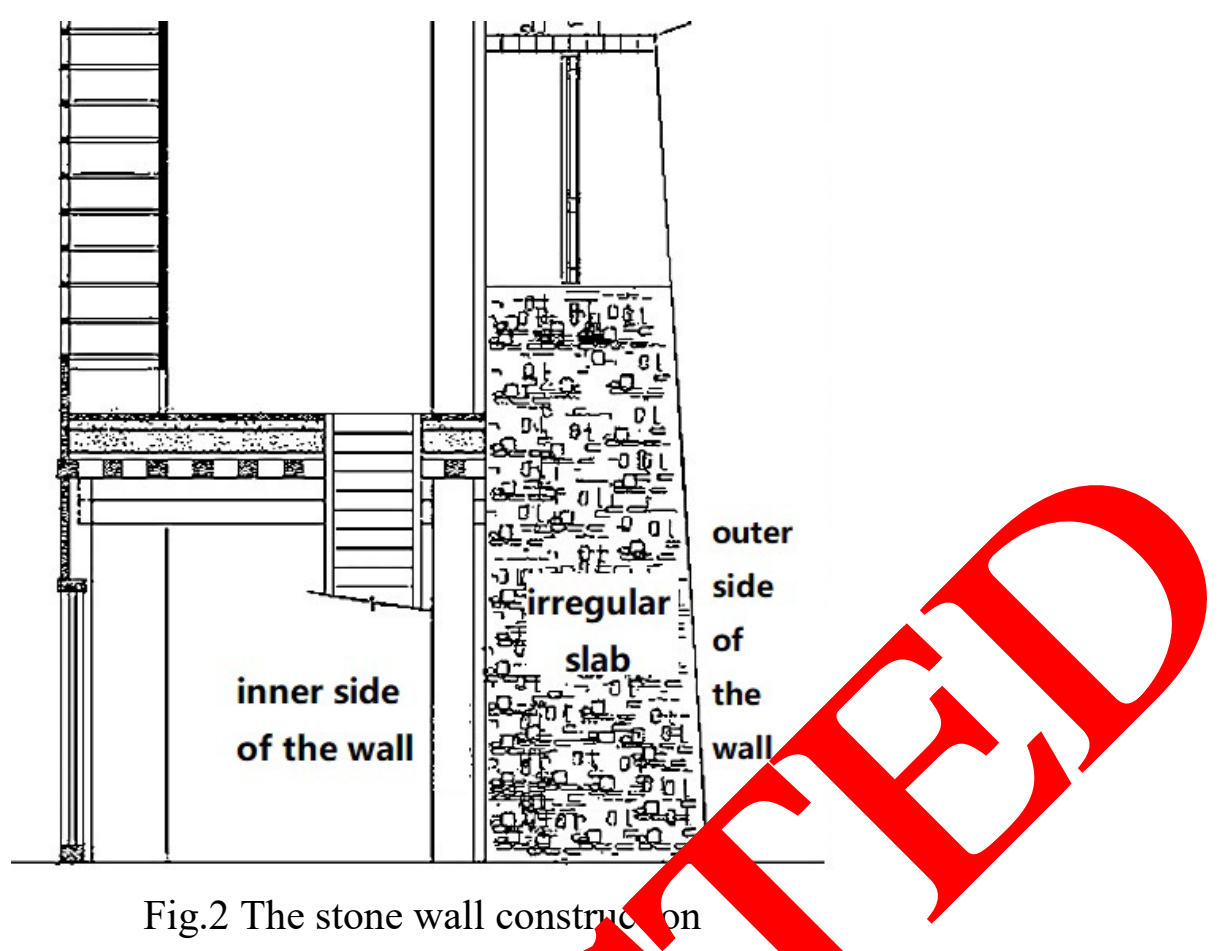

In addition to the flake-stone walls, many buildings of Labrang Monas $/$ use rammed soil walls and brick walls. The rammed soil walls are generally $\mathrm{u}$ ed in walls of courtyard and walls of Monk houses. The rammed soil walls in Labrang Monastery $u$. the yellow andstone soil of Xiahe County, and the thickness of the wall is generally between 0.5- In te ms of color, the inner and outer sides of rammed soil walls are painted into whit dor yellow -1 19. 3). Besides, the brick wall can be found in the Han-style sleeping palace of Jiam yarrs The wall is made of black brick, and its construction is from the Han-style of Qing Dynas (Figul 4 )

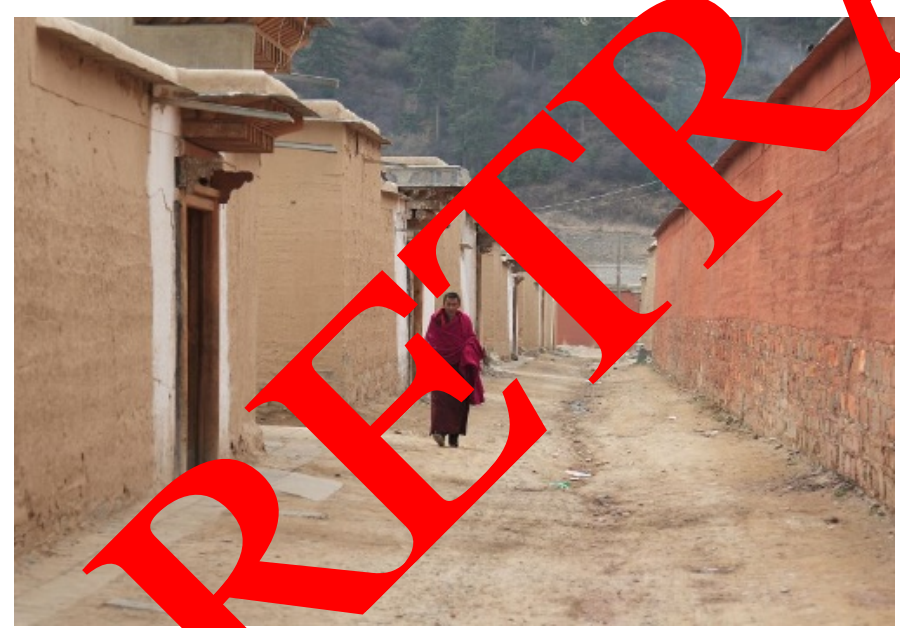

19.3 Rammed soil wall

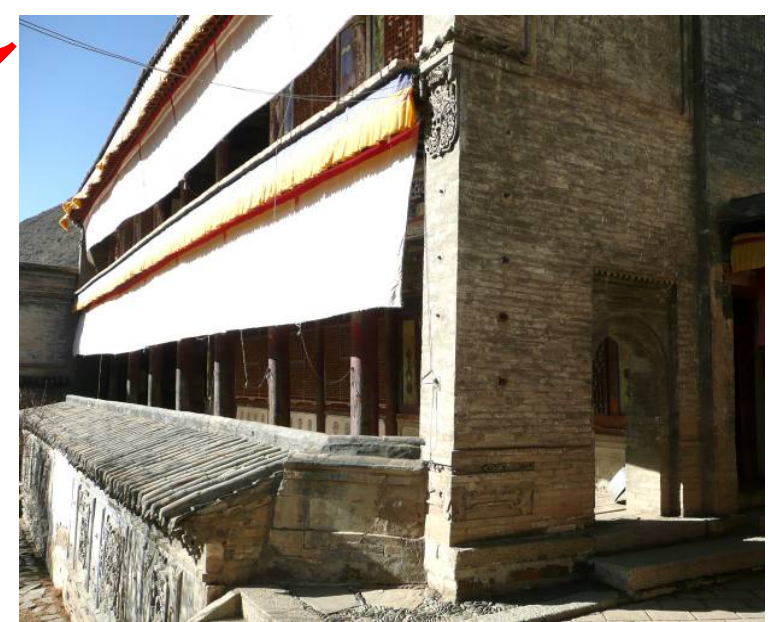

Fig.4 Brick wall

(2) the dasiphora wall construction.

The dasiphora wall is a decorative part on the important buildings of the Labrang Monastery. They're usually used on the exterior walls, with the function of anti-theft and protecting the walls from being washed away by rain. The dasiphora walls are usually painted in red. The dasiphora wall of the border is to bundle the red wicker into small bundles, dyed brown, dark brown and then cut, and the roots are densely arranged on the wall (Fig. 5). The upper and lower sides of the dasiphora wall are covered with decorative wooden strips and small picking heads. The wooden strips have vertical rods, and the middle is fixed with vertical wooden strips. Add a horizontal tiebeam on the top and bottom of the dasiphora wall, and a row of short woods is laid under the top and bottom tiebeam (Fig. 6). The 
construction of the entablature is to placing slab on the tiebeam to make the cornice. The dasiphora wall is decorated with gilded bronze plates which has patterns of Buddhist and Sanskrit[8]. The construction of a dasiphora wall from the bottom is "square rafter - tiebeam - slab- dasiphora tied up with string - tiebeam - rafter - copestone" in proper sequences (Figure 7).
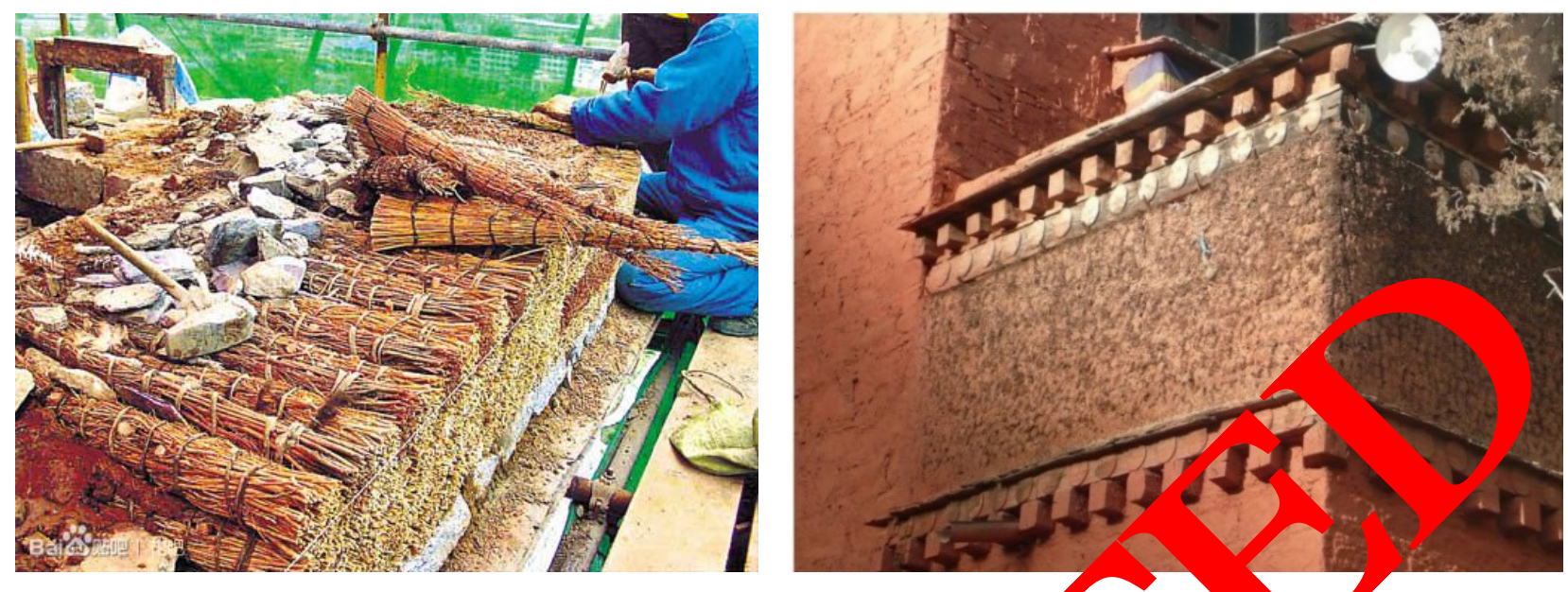

Fig.5 The dasiphora bundled and placed on the wall
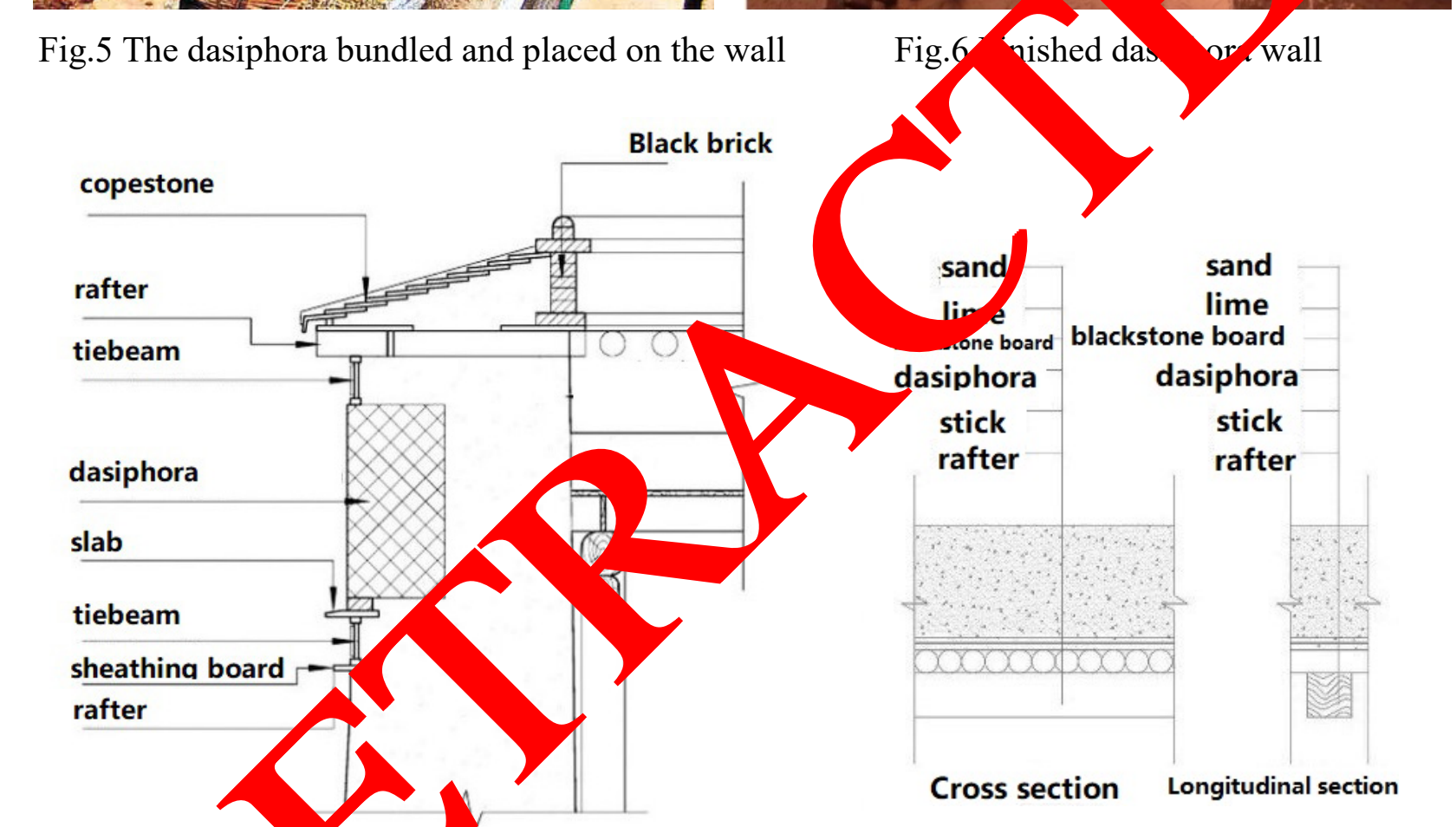

2.1.2 foc co dauction

Fig. 8 The Tibetan-style roof construction

The is an-styru roof construction of the Labrang Monastery (Fig. 8) can be divided into six layers. From ttom to top, it is wood rafters, sticks, dasiphora, blackstone boards, lime and sand. In comparison, the thickness of the sand layer is relatively thick, generally between $45 \mathrm{~cm}$ and $55 \mathrm{~cm}$. There is a scattered water slope in the sand layer, and the slope of the scattered water is generally $2 \%$. This construction is relatively simple and cheap, so it is widely used in the roof construction of Labrang Monastery.

\subsection{Building Materials}

The building materials used in the monastery mainly include soil, stone and wood, according to the research.

(1) Material of soil

According to the investigation, the soil used in buildings can be divided into black soil, loess and yellow-sand-stone-soil, which are all from the local site. The black soil is generally used as bonding 
material due to its good adhesion ability, and it needs to be soaked with water before use. Because of the soft texture, the loess is usually applied to the inner walls of the building for drawing murals, but it is usually mixed with a certain proportion of fine sand to use. The yellow-sand-stone-soil is often used as the roof material. The general construction is to lay a branch of about $50 \mathrm{~cm}$ on the rafter of the roof, then make a layer of cobblestone about $60 \mathrm{~cm}$ thickness above[9]. The craftsmen filled the gap with black soil and made the layer of black soil's thickness up to $50 \mathrm{~cm}$, then filled the yellow-sand-stone-soil on it and continue to tamp. (Fig.9).

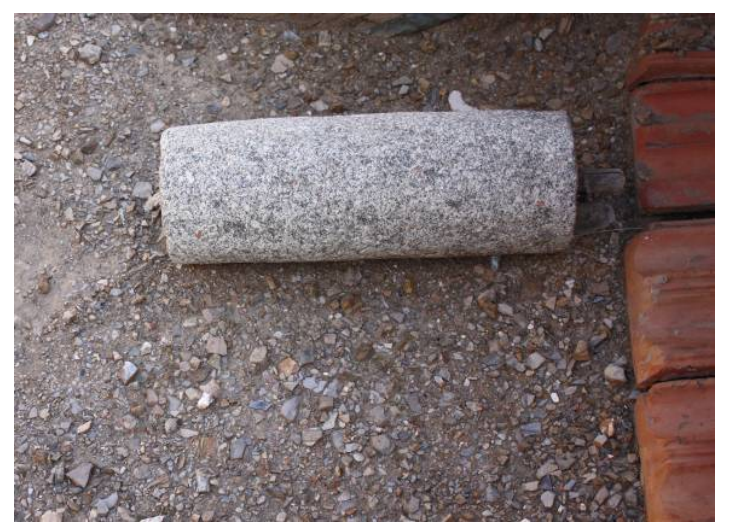

Fig.9 Roll-over tool of roof

\section{(2)Material of stone}

The construction of Labrang Monastery uses a load-bearing system. It is generally divided into two normal size of block stone is $17 \mathrm{~cm} * 23 \mathrm{~cm} * 35 \mathrm{~cm}$. The th

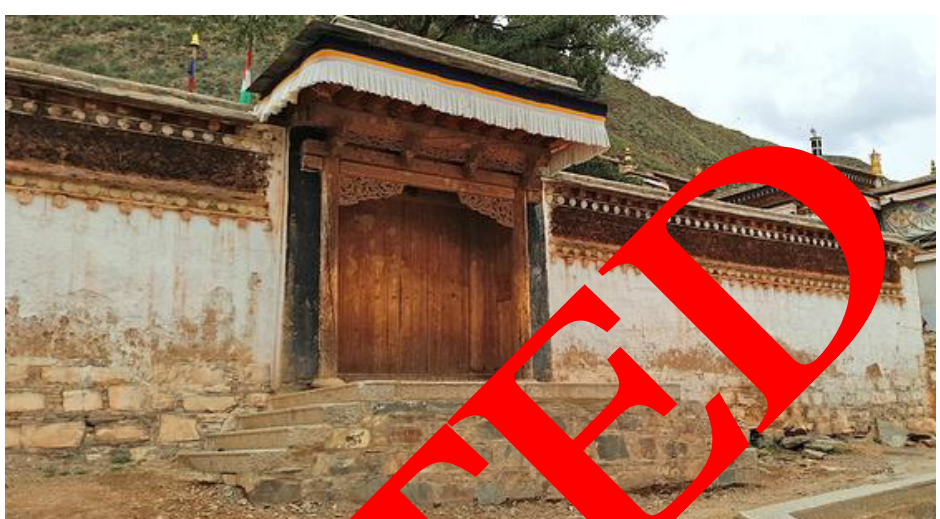

Fig. 10 tono the botto $n$ of the wall which is used to level or to fill the gap of block ane. (Fig.

(3)Material of wood

The interior construction of Labrang Monaste ir many made of wood. Wood is mostly spruce, in addition to columns, beams, sparr buckets rafters, etc. used in building supporting frames, while also used in door railings, w dow imes an yterior floors (Fig. 11).

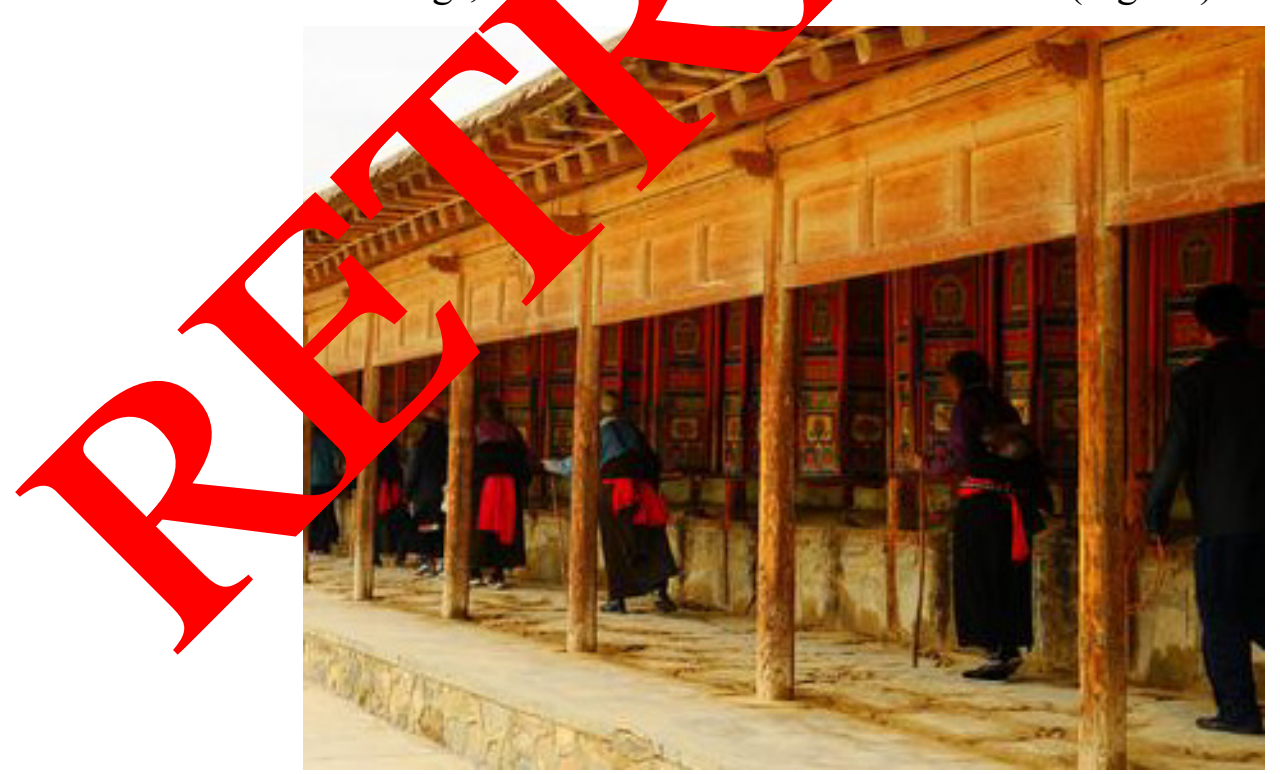

Fig.11 Wood Use in Labrang Monastery

In addition, the building materials of Labrang Monastery include bricks, which only cover a small area. Only the summer residential house in Jiamuyang Living Buddha Palace is built with clear water bricks, and the decorative patterns on the bricks are exquisitely carved. 


\section{Analysis of the Physical Environment of Labrang Monastery}

\subsection{Architectural acoustic environment}

Labrang Temple is located in the west of county town, where far from the downtown area. The temple is surrounded by mountains and rivers on three sides, so it is relatively independent and low external noise harrasment[10]. The materials and construction make the stone-soil wall in the monastery very thick that the walls are usually more than $1 \mathrm{~m}$ thick. In addition, there are few small windows on the walls, usually there is little noise in the room, which provides a good sound environment for monks to practice and study.

The average noise pressure level of sutra hall in the monastery is $\mathrm{L}=24.8 \mathrm{~dB}(\mathrm{~A})$ by measuring the Kalachakra academy and Hevajra academy. When 25 monks recite sutras indoor, the equivalent continuous sound pressure level of Kalachakra academy is Leq=68.3 $\mathrm{dB}(\mathrm{A})$, whic is in the appropriate sound pressure level for the talking sound environment.

\subsection{Building Optics Environment}

The control and application of light are very important in religious chitactures general indoor lighting standards, Labrang Temple, as a religious buildi its li oling in ironment construction requirements not only meet the site function but also mee speer thaking the Great Sutra Chanting Hall of Labrang Monastery as an example, th space cen $1 \mathrm{f}$ " Se Sutra Hall is partly connected with the second floor, and the high side sk igh set on th second floor for daylighting. The overall illumination of the building is very lo the sk ht mainly provides the key illumination for the northern rostrum and the Buddhist ni ne, so as to vist. $y$ strengthen and reflect the central position of the sacred space in the interior. In ddition, the matefial and construction of the skylight are worth mentioning in the construction of in or light eny ronment. Flat skylights are set up on the north side of the roof of Kalachakra academy' ditoriv 1 . The skylights are fixed on the roof with angle steel brackets. Not all sunken sk hts on thervor are such daylighting skylights. The sunken skylights on the south sides of Kala takry demy's roofs are directly open, that is, daylighting and ventilation and heat dissipation. 1 i e are also many high-side skylights, with a thin layer of light-colored paint on ordina $s$, whic produces uniform diffusion and penetration. The radiation effect effectively avoids glan problem caused by direct sunlight around noon time.

The interior overall lighting or ong Monastery's Sutra Halls is of low brightness. It only lights and illumine es the $\mathrm{B}$, ha statues and niches. This practice not only creates a mysterious religious atm sp but also ays a symbolic role of darkness with Buddha's guidance, and achieves a high deg. e of $\mathrm{u}$. of content and form.

\subsection{Building therr al en ironment}

The natural iron of Labrang Monastery is dry-hot climate, with strong solar radiation, large temperature erence setween day and night, and few rainfall. Academic buildings and Buddhist stery gs are two main types of buildings in Labrang Monastery, which are public uildin $s$ in term of use. Their requirements for building thermal insulation are not high, but their ma astruction methods do reflect the characteristics of energy saving and comfort.

Most o materials used are ecologically recyclable, such as natural stones, raw soil, timber, margin grass, $c$. The roof of stone-soil mixed construction wall and civil mixed construction with thickness more than $1 \mathrm{~m}$ ensures good thermal performance of the building, making it warm in winter and cool in summer. Spring, summer and autumn are hot seasons in Gannan area. As a good heat storage body, the thick walls absorb a large amount of solar radiation heat during the day. Because of the delayed effect, it does not radiate until night, which ensures that the indoor environment is in a cool state during the day when it is used in high frequency. The thermal comfort effect of winter buildings is not as obvious as that of summer buildings, because the main hall buildings need to open doors in most cases, and the sealing of wood windows is poor, some skylights do not contain glass, so there is not much difference between indoor and outdoor's temperature. In winter, the indoor thermal environment of the Sutra Hall and Buddhist Hall is stable, in a state of no wind or fresh air, which is conducive to keep awake and quick thinking when monks concentrate on reciting sutras. 
Labrang Monastery building does not need air conditioning in summer or heating in winter (mainly refers to academic buildings and Buddhist halls). The above thermal environment effect can be obtained only through materials and construction. The cost is low, and the ecological environment protection is high-performance[11]. This has certain enlightenment for the protection and inheritance of Tibetan Buddhist buildings and even for the design of public buildings.

\section{Conclusion}

The building materials and construction of Labrang Monastery have great influence on the acoustics, optics and thermal environment of the building. From the perspective of architectural technology, this paper interprets the architecture of Labrang Monastery, which not onlr-nrovides more references for the protection and renovation work, but also triggers some re rections the design of modern religious architectures.

1)Location:

On the view of acoustic environment control, we should avoid densely po pulated area d noose quiet places with good natural environment;

2)Space layout:

From the perspective of optics environment, religious spaces hould no $\mathrm{ly}$. onform to the functions of religious activities, but also to meet the spiritual $\mathrm{n}$ eds, to stren anen the grasp and utilization of natural lighting;

3)Building energy efficiency:

We should make the best use of adjusting and desig ing the thermal environment of the interior space via physical means so as to make the buildings w "free breat 1 " and ecologically sustainable.

\section{Acknowledgements}

This paper is supported by the National Natura S Ience, oundation of China(NO. 51568038), the National Natural Science Foundation ina, (No 51608421) and the PhD Foundation of Weifang University(NO. 2018BS13)

\section{References}

[1] Xinya Xu. Trad ona betan R rigious Architecture of Labuleng-Monastery, J. World Architecture.

[2] Yunying P n, Qi o. The ląndscape pattern analysis of Labuleng Temple, J. Architecture and Culture. 70 1). $50-51$.

[3] Jiar an. S Aode analysis of religious architecture in Inida, J. Journal of Xi'an vivers $y$ of Arc recture and Technology. 12 (2002)379-382.

[4] Lin Ar. muan Qinwang of Qinghai and Labrang monastery, J. Journal of Qinghai normal unive, (philosophy and social sciences), 39(2017)83-86.

[5] Blobzang Tshering. A textual research on the relationship between the $9^{\text {th }}$ Panchen and Labrang Lamasery, J. Tibetan Studies, 2(2017)24-30.

[6] Qi Gao, Yunying Ren. The study of space model about the building of sutra chanting hall of Tibetan Buddhism temple in Gannan area, J. Journal of Xi'an University of Architecture \& Technology(Natural Science Edition). 50(2018) 78-84

[7] Meji Zhuo. A brief introduction to the Labrang event, J. Journal of Sichuan Minzu College. 26(2017)41-45.

[8] Zhihe Guo. Urbanization study on the type of business around temples in the Tibetan area, J. Journal of Tibet University. 30(2015)103-113. 
[9] Zhiwei Zou, Yongjian Hou. Monk's system of labrang temple and ecological environment in the south Gansu Tibetan area during the period of Republic of China, J. Northwest Population. 33(2012)93-97

[10] Dpav-ris-don-grub. The relationship of dharma between four Gulug temples in Tibet and Labolung temple in Gansu, J. Journal of Tibet national institute (philosophy and social science). 31(2010)30-38.

[11] Ping Zhang, Xinya Xu, Hua Chen. Brief analysis of building special layout in Labuleng temple, J. Shanxi Architecture. 34(2008)52-53.

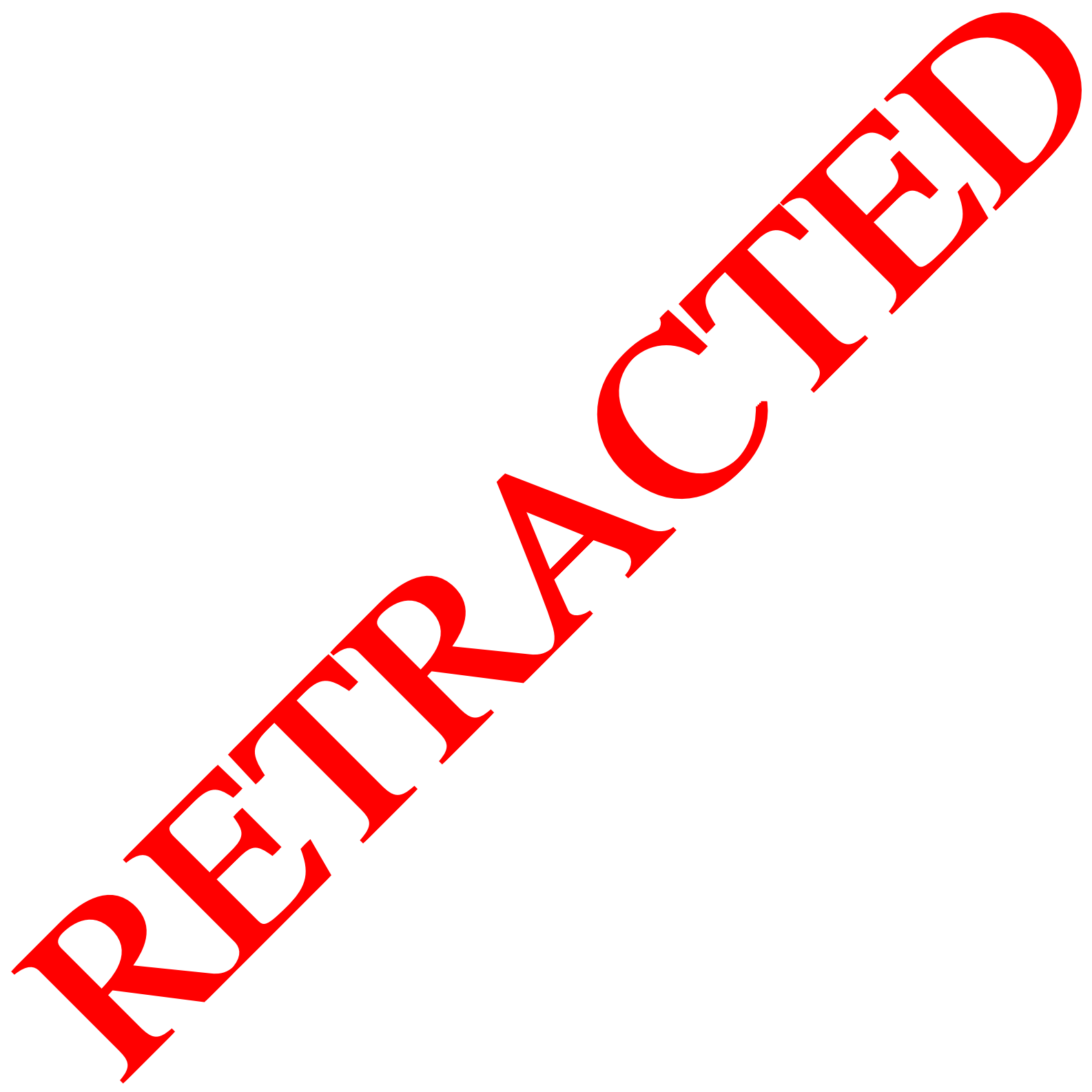

